\title{
Maximum size of drops levitated by an air cushion
}

\author{
Jacco H. Snoeijer, ${ }^{1,2}$ Philippe Brunet, ${ }^{3}$ and Jens Eggers ${ }^{1}$ \\ ${ }^{1}$ Department of Mathematics, University of Bristol, University Walk, Bristol BS8 1TW, United Kingdom \\ ${ }^{2}$ Physics of Fluids Group and J. M. Burgers Centre for Fluid Dynamics, University of Twente, \\ P.O. Box 217, 7500 AE Enschede, The Netherlands \\ ${ }^{3}$ Laboratoire de Mécanique de Lille, UMR CNRS 8107, Boulevard Paul Langevin, 59655 Villeneuve d'Ascq Cedex, France
}

(Received 2 September 2008; revised manuscript received 11 February 2009; published 18 March 2009)

\begin{abstract}
Liquid drops can be kept from touching a plane solid surface by a gas stream entering from underneath, as it is observed for water drops on a heated plate, kept aloft by a stream of water vapor. We investigate the limit of small flow rates, for which the size of the gap between the drop and the substrate becomes very small, to obtain a full analytical description of stationary drop states and their stability. Above a critical drop radius no stationary drops can exist, below the critical radius two solutions coexist. However, only the solution with the smaller gap width is stable, the other is unstable. We compare to experimental data and use boundary integral simulations to show that unstable drops develop a gas "chimney" that breaks the drop in its middle.
\end{abstract}

DOI: 10.1103/PhysRevE.79.036307

PACS number(s): 47.55.nb, 47.55.D-

\section{INTRODUCTION}

Drops levitated on an air cushion have numerous applications, and have for a long time generated interest. For example, in lens manufacture drops of molten glass can be prevented from contact with a solid substrate [1]. This is achieved by levitating the glass above a porous mould, through which an air stream is forced. A second example is the so-called "Leidenfrost" drop [2], a drop of liquid on a plate hot enough to create a film of vapor between the drop and the plate [3-6]. Since the drop is thermally insulated by the vapor film, it can persist for minutes [5]. Finally, a thin air film is believed to play a crucial role for the "noncoalescence" of a liquid drop bouncing off another liquid surface [7-9].

The question we will address in this paper is whether for a given set of parameters, in particular the radius of the drop as it "rests" on the substrate, a stationary solution exists and whether it is stable. Apart from lens manufacture [1], this question is important for the manipulation of corrosive substances [10] or the frictionless displacement of drops [6]. Of particular interest is the maximum drop size that can be sustained, and the limit of very small flow rates. The drop continues to levitate in this limit since the gap between the liquid and the substrate becomes very small, so the lubrication pressure produced by the viscosity of the gas becomes significant. This enables us to employ asymptotic methods, making use of the disparity of scales between the gap size and that of the drop.

Experimentally, it is observed that the stability limit is reached when the radius equals at least a few capillary lengths $\ell_{c}=\sqrt{\gamma /(\rho g)}$. This natural length scale for our system is determined by the surface tension $\gamma$, density $\rho$ of the liquid, and acceleration of gravity $g$. At a few capillary lengths, the drop is flattened to a pancake shape. Biance et al. $[5,11]$ observed a critical radius

$$
\frac{r_{\max }}{\ell_{c}}=4.0 \pm 0.2
$$

where $r_{\max }$ is defined in Fig. 1. Beyond this radius, "chimneys" appeared, i.e., bubbles of air trapped below the curved and concave surface of the drop, that rise owing to buoyancy and eventually burst through the center of the drop. This suggests that the critical radius is related to the RayleighTaylor instability of a heavy fluid (the drop) layered above a light fluid (the gas layer). In $[5,11]$ this idea is used to estimate $r_{\max } / \ell_{c} \approx 3.83$.

While this is close to the experimental value, the argument ignores the gas flow responsible for the levitation force. This flow was taken into account by Duchemin et al. [1], who calculated the static shape of a drop levitated above a curved porous mould, using a combination of numerics and asymptotic arguments. For large enough drop volume, they found no physical solutions, while for smaller drops multiple solutions were calculated numerically. This work was complemented recently by a numerical stability analysis [12].

A large number of studies of Leidenfrost drops have focused on the appearance of self-sustained oscillations of the drop [3,5,13-18]. These oscillations can sometimes lead to a morphological bifurcation of the drop, which takes the shape

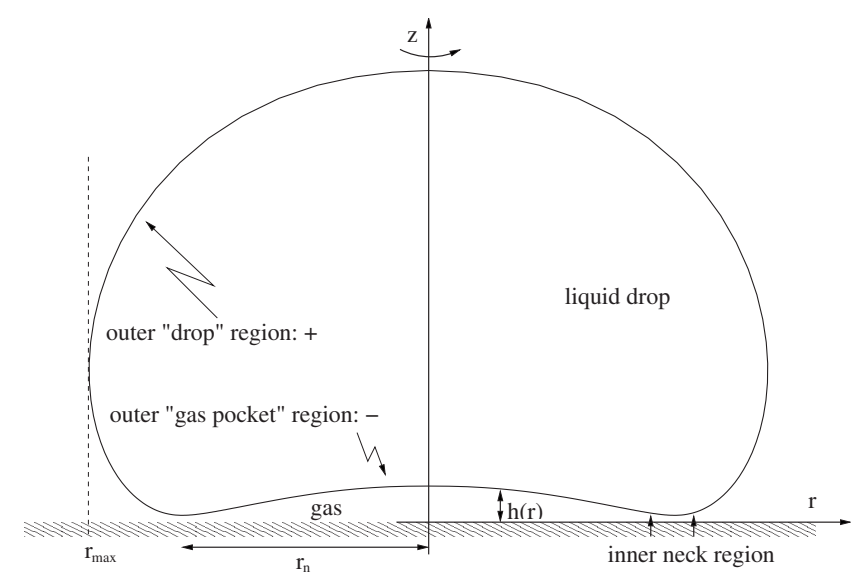

FIG. 1. Definitions and sketch of the matching regions. The liquid-gas interface will be denoted by $z=h$. In the "gas pocket" region below the drop, we can write $h$ as a function of the radial coordinate $r$. Due to the overhang exterior of the neck, $h(r)$ is multivalued in the "drop" region. 
of a star $[3,14,15,17,18]$. Similar star-shaped drops have been reported in drops vertically vibrated on nonsticky surfaces, and the shape is generally attributed to a parametric instability [19]. Our original question was whether oscillations could perhaps be explained even in the limit of viscous drops, which we focus on in this paper. This is not the case, since both our asymptotic results and simulations of the complete dynamics (i.e., beyond linear stability analysis) show that once unstable, a drop breaks up owing to the formation of a chimney.

We treat both the liquid drop and the surrounding gas in the inertialess (Stokes) limit. For the asymptotic analysis, we also require the drop to be much more viscous than the gas. The main effect of this assumption is that there is hardly any flow inside the drop, so it can be treated as being in hydrostatic equilibrium at any instant in time. We also prescribe the rate at which gas is injected into the underside of the drop, thus ignoring the possible interplay between drop dynamics and vapor production in the Leidenfrost problem.

Our analysis is similar in spirit to the earlier paper of Duchemin et al. [1], but we only address the simpler case of a flat substrate. As a result, we are able to perform all the calculations analytically (up to a few universal constants, which have to be computed numerically). Our solution curves are in qualitative agreement with those for a curved substrate $[1,12]$, but now imply a full analytical description. In addition, we determine the stability boundary of the stationary states. We find the maximum stable radius

$$
\frac{r_{\max }}{\ell_{c}} \approx 4.35-\tilde{r},
$$

where $\tilde{r}$ goes to zero in the limit of vanishing gas flow.

For typical experimental flow rates we find that $\tilde{r} \approx 0.4$, consistent with the experimental result (1). At the end of the paper, we discuss how our analysis relates to the stability argument of $[5,11]$, based on the Rayleigh-Taylor instability.

\section{PROBLEM FORMULATION}

\section{A. Geometry and dimensionless parameters}

We consider axisymmetric drops of liquid, levitated above a flat surface by gas flowing into the underside of the drop; cf. Fig. 1. We set out to find the shape of stationary drops and their stability, as a function of the gas flow rate and the drop volume. The size of the drop is expressed by the Bond number

$$
\mathrm{Bo}=\frac{R^{2}}{\ell_{c}^{2}},
$$

where $V$ is the volume of the liquid drop, and $R$ $=(3 V / 4 \pi)^{1 / 3}$ is the unperturbed radius. The dimensionless gas flow rate supporting the drop reads

$$
\Gamma=\frac{Q \eta_{\mathrm{gas}}}{\ell_{c}^{2} \gamma},
$$

where $Q$ is the volume of gas that escapes through the narrow neck region (see Fig. 1) per unit of time, and $\eta_{\text {gas }}$ is the viscosity of the gas. Our analysis will identify the flux $Q$ as the relevant quantity, which can be calculated by integrating the gas flux entering from underneath up to the neck position $r_{n}$. Let us also introduce a slightly different dimensionalization of the flow rate,

$$
\chi=\frac{6 \Gamma \ell_{c}}{\pi r_{n}}=\frac{6 Q_{n}}{\pi r_{n} \ell_{c}},
$$

which will appear naturally in the analysis.

Finally, another parameter is the viscosity ratio between liquid and gas,

$$
\lambda=\frac{\eta_{\text {drop }}}{\eta_{\text {gas }}},
$$

but which will be considered asymptotically large for most of this paper. Throughout, lengths will be expressed in $\ell_{c}$, velocities in $\gamma / \eta_{\text {gas }}$, and stresses in $\gamma / \ell_{c}$.

\section{B. Structure of the problem}

The problem we attempt to solve is the inertialess, axisymmetric fluid flow equations, with a prescribed influx of gas into the underside of the drop. Most of our analytical work assumes in addition that the drop is much more viscous than the gas. The structure of the expected solution is shown in Fig. 1. The gas pressure below the drop has to be sufficiently large in order to support the weight of the drop. In the limit of small dimensionless gas flux $\Gamma$, the gap between the drop and the substrate must therefore be small in order to generate enough pressure. The underside of the drop inflates to a gas pocket, whose width is of similar size to the drop itself. The narrow gap is formed in a small neck region only, where a large curvature assures that the gas pressure can be sustained by corresponding surface tension forces. Apart from this viscous neck region, the gas pressure is constant, both in the gas pocket as well as to the exterior of the neck.

This leads to the following asymptotic structure of the problem, characterized by the matching between three different regions. In the limit of small flux, all viscous effects become localized in a small neck region, situated at a radius $r=r_{n}$ from the center. In this region, there exists a balance between viscous and surface tension forces. In addition, the slope of the gap profile $h(r)$ turns out to be small in this region, so lubrication theory [20] permits to reduce the flow equations to an ordinary differential equation for $h(r)$. We will call this the inner solution or neck region.

To close the problem, boundary conditions are needed. These are provided by two outer regions on either side of the neck, denoted by "-" (the gas pocket toward the center of the drop) and "+" (the outside of the drop). Both regions are controlled by a balance of gravity and surface tension alone. First, we solve the equations in each of the regions individually. Second, we require that both the slope and the curvature of the profile match smoothly at the boundaries between two regions. This leads to a set of equations that determines stationary drop solutions uniquely. Solutions exist only below a certain critical neck position $r_{c}$, in which case we find two branches, one with a small gap width (the lower branch) and an upper branch with a larger gap width. 
Our stability analysis of the two branches is based on the observation that the relevant dynamic variable is the position $r_{n}$ of the neck, which can shift easily. The maximum stable neck radius does not coincide with $r_{c}$, but is significantly smaller, located on the lower branch. We show that this point corresponds to the drop of maximum volume, consistent with the numerical results by [12].

\section{INNER SOLUTION: NECK REGION}

\section{A. Lubrication approximation}

We consider incompressible, axisymmetric flow in the gas layer, so that mass conservation gives

$$
r \dot{h}+(r h \bar{u})^{\prime}=r v(r) .
$$

Here $\bar{u}$ is the depth-averaged horizontal velocity of the air layer, while $v(r)$ is the rate at which air volume is injected per unit area below the drop. The main focus of the paper will be on stationary states and their stability. Stationary drop profiles are found by taking $\dot{h}=0$, and integrating Eq. (7) to

$$
r h \bar{u}=\frac{\Gamma(r)}{2 \pi},
$$

where $\Gamma(r)=2 \pi \int_{0}^{r} d r^{\prime} r^{\prime} v\left(r^{\prime}\right)$ is the flux in the lubrication layer. In the case in which the injection source is localized at $r=0$, the flux $\Gamma$ is simply constant.

To get a closed equation for $h(r)$ in the neck region, we solve for $\bar{u}$. As our results will confirm, the neck region is shallow, $h^{\prime} \ll 1$, meaning that we can use the lubrication approximation [20] to analyze the flow; see Fig. 1. Owing to the large viscosity ratio between the drop and the surrounding gas, the liquid drop acts as a no-slip boundary, and the flow in the gas layer is well approximated by

$$
u=6 \bar{u}\left(\frac{z}{h}-\frac{z^{2}}{h^{2}}\right) .
$$

Since the Reynolds number is very small in typical experiments, we use the Stokes approximation [20] to relate this velocity to the pressure. As there is almost no flow inside the drop, the liquid will be at hydrostatic equilibrium, $p_{\text {liquid }}$ $=p_{0}-z$. At the liquid-gas interface, the pressure thus equals $p_{0}-h$ at the interior of the drop. Furthermore, the pressure jump across the interface equals the curvature times the surface tension, so one obtains the lubrication pressure inside the gas layer as

$$
p=p_{0}-h-h^{\prime \prime} .
$$

In what follows, we will show that the width of the neck region scales as $\Gamma^{1 / 5}$ and thus is asymptotically small in the limit of vanishing flux. We are therefore permitted to neglect the axisymmetric contribution to the curvature in the neck region. Using the horizontal component of the Stokes equation, $p^{\prime}=\partial^{2} u / \partial z^{2}$, we find

$$
\bar{u}=\frac{1}{12} h^{2}\left(h^{\prime}+h^{\prime \prime \prime}\right) .
$$

Now Eqs. (8) and (11) provide a closed equation for the stationary interface profile $h(r)$,

$$
h^{3}\left(h^{\prime}+h^{\prime \prime \prime}\right)=\frac{6 \Gamma(r)}{\pi r} .
$$

The right-hand side of Eq. (12) represents the viscous stress in the flow, and will only become important when $h$ is small, i.e., in a small neck region around $r_{n}$, where we may set $r=r_{n}$. This gives

$$
h^{3}\left(h^{\prime}+h^{\prime \prime \prime}\right)=\chi
$$

with

$$
\chi \equiv \frac{6 \Gamma\left(r_{n}\right)}{\pi r_{n}} .
$$

A crucial observation is that there is no need to know the precise form of how the gas is injected, but one only requires the flux across the neck. This of course provides a great simplification for the Leidenfrost problem, where evaporation rates are related in a complicated way to the temperature profile inside the drop.

\section{B. Similarity solution for the neck region}

As gravity is unimportant in the thin neck region, Eq. (13) can be further simplified to

$$
h^{3} h^{\prime \prime \prime}=\chi .
$$

Since we are interested in the limit of small flux, we look for similarity solutions

$$
h(r)=\chi^{\alpha} H(\xi), \quad \text { where } \xi=\frac{r-r_{n}}{\chi^{\beta}}
$$

giving

$$
H^{3} H^{\prime \prime \prime}=1, \quad \text { with } 4 \alpha-3 \beta=1 .
$$

Note that this same equation emerges in film drainage problems during droplet coalescence [21] and sedimentation [22].

In the limit $\xi \rightarrow \infty$, the solutions have to match onto a sessile drop of constant curvature. Since

$$
h^{\prime \prime}=\chi^{\alpha-2 \beta} H^{\prime \prime},
$$

one requires that $\alpha-2 \beta=0$ for the curvature to remain finite as $\chi \rightarrow 0$. Together with Eq. (17) this fixes $\alpha=\frac{2}{5}$ and $\beta=\frac{1}{5}$, and hence we have

$$
h(r)=\chi^{2 / 5} H\left(\frac{r-r_{n}}{\chi^{1 / 5}}\right) .
$$

The form of the similarity function will be determined from the matching below. The fact that $\alpha>\beta$ justifies the assumptions made so far. First, we find that $h^{\prime} \ll 1$ in the limit $\chi \rightarrow 0$, justifying the use of lubrication theory. Similarly, $h^{\prime} \ll h^{\prime \prime \prime}$, so that both gravity and the axisymmetric curvature can indeed be neglected in the neck region.

The asymptotic behavior of Eq. (17) is quadratic for both $\xi \rightarrow \pm \infty$,

$$
H_{+}=\frac{1}{2} K_{+} \xi^{2}+S_{+} \xi \quad \text { for } \xi \rightarrow \infty,
$$




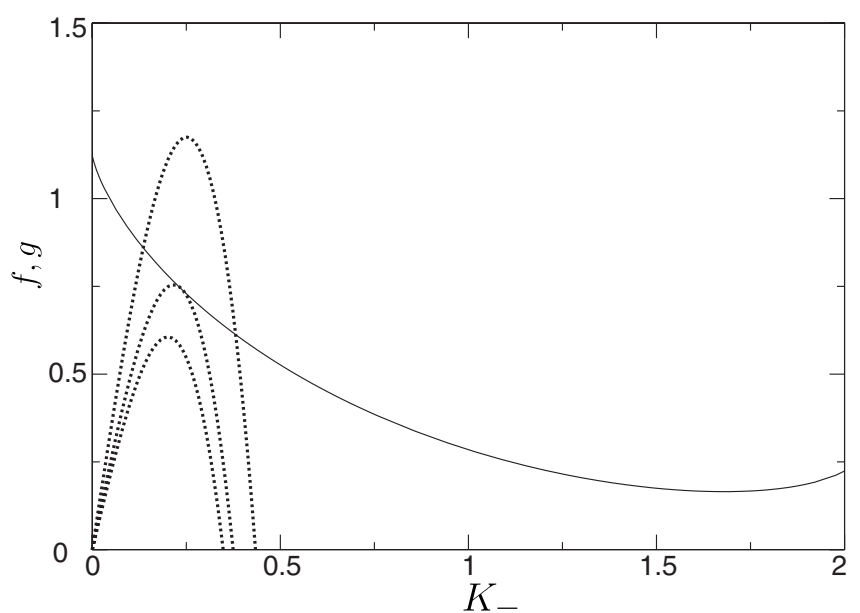

FIG. 2. Solid line: the function $f$ relates the slope $S_{-}$to curvature $K_{-}$of the inner solution [Eq. (23) with $K_{+}=2.17$ ]. Dotted lines: the function $g$ provides the matching condition between inner region and gas pocket region [Eq. (51) with $\chi=10^{-7}$ ]. The three curves correspond to values $r_{n}=3.55$ (below critical), $r_{n}=3.62$ (critical), and $r_{n}=3.65$ (above critical).

$$
H_{-}=\frac{1}{2} K_{-} \xi^{2}+S_{-} \xi \quad \text { for } \xi \rightarrow-\infty .
$$

Physically, the values of the asymptotic curvatures $K_{ \pm}$set the pressure in the corresponding outer regions.

Since Eq. (17) is of third order, solutions can be specified by three independent parameters, one of which can be absorbed into a shift of $\xi$. Therefore, the two asymptotic curvatures $K_{ \pm}$uniquely determine the solution. As a consequence, the slopes $S_{ \pm}$are dependent variables. To perform the matching, we require the function

$$
S_{-}=-f\left(K_{-}, K_{+}\right) \text {, }
$$

whose existence is assured by the above argument. Since Eq. (17) is invariant under the transformation $H \rightarrow H / a$ and $\xi$ $\rightarrow \xi / a^{4 / 3}$, one must have

$$
f\left(K_{-}, K_{+}\right)=K_{+}^{1 / 5} f\left(\frac{K_{-}}{K_{+}}, 1\right),
$$

where we used $a^{5 / 3}=K_{+}$. This scaling was already mentioned in [21] This function is computed numerically and is plotted in Fig. 2. We show below that stationary solutions correspond to the intersection of $f$ with another function $g$, shown in the same figure. It can be seen that the matching breaks down at a critical neck radius $r_{n}$, beyond which stationary solutions cease to exist.

\section{OUTER SOLUTIONS}

Having seen that viscous effects are localized in the neck region, the rest of the drop is at static equilibrium. Hence, the pressure is constant both in the gas pocket between the drop and the substrate, as well as to the exterior of the neck. These pressures are not equal, however, since one requires a pressure difference to drive the flow across the neck. In Fig. 1, we therefore distinguish two outer regions, denoted by +

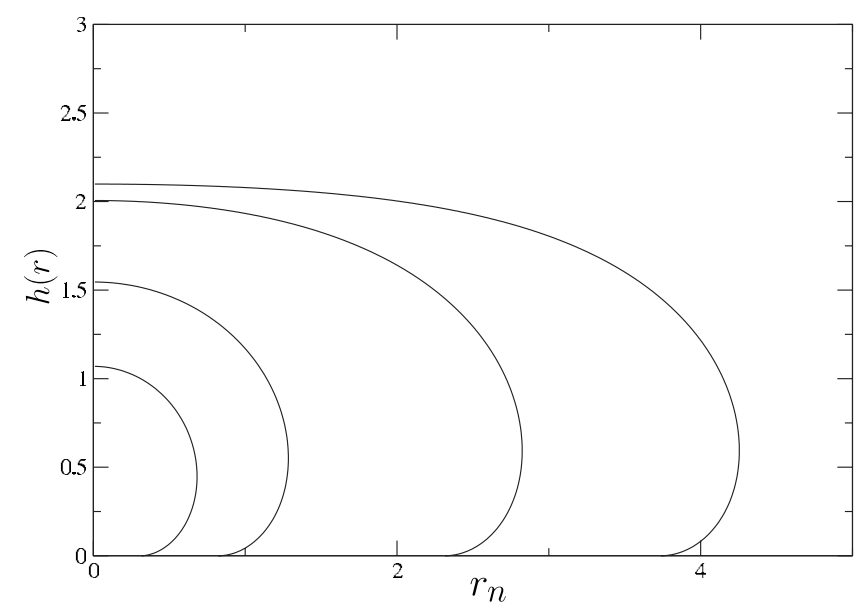

FIG. 3. The outer solution of the "drop" region corresponds to a perfectly nonwetting sessile drop. The size of the drop, characterized by $r_{n}$, sets the curvature $K_{+}$for the inner solution.

and - , respectively. Since $p_{ \pm}=p_{0}-h-\kappa$, the outer solutions can be obtained from

$$
\kappa+h=c_{ \pm},
$$

where $\kappa$ is the curvature of the interface. The constants $c_{ \pm}$ determine the pressure difference across the neck,

$$
\Delta p=p_{-}-p_{+}=c_{+}-c_{-},
$$

and will follow from the matching.

\section{A. Outer "drop" region: +}

Below we will find that the profile of the "drop" region requires $d h / d r \rightarrow 0$ as $h \rightarrow 0$ in order to match to the neck smoothly. This corresponds to a perfectly nonwetting sessile drop (Fig. 3). When matching the curvature, we also require $d^{2} h / d r^{2}=K_{+}$as $h \rightarrow 0$. Owing to the vanishing slope near $h$ $=0$, we are allowed to write $\kappa=d^{2} h / d r^{2}$ in Eq. (24). Hence, one finds $c_{+}=K_{+}$.

To deal with the overhang of the sessile drop, it is convenient to solve the profile in terms of the arclength $s$ along the interface. We define $\theta$ as the angle with the horizontal and rewrite Eq. (24) as

$$
\begin{gathered}
\frac{d \theta}{d s}=-\frac{\sin \theta}{r}-h+K_{+}, \\
\frac{d h}{d s}=\sin \theta, \\
\frac{d r}{d s}=\cos \theta,
\end{gathered}
$$

with boundary conditions

$$
\begin{aligned}
& \theta(0)=0, \\
& h(0)=0, \\
& r(0)=r_{n},
\end{aligned}
$$




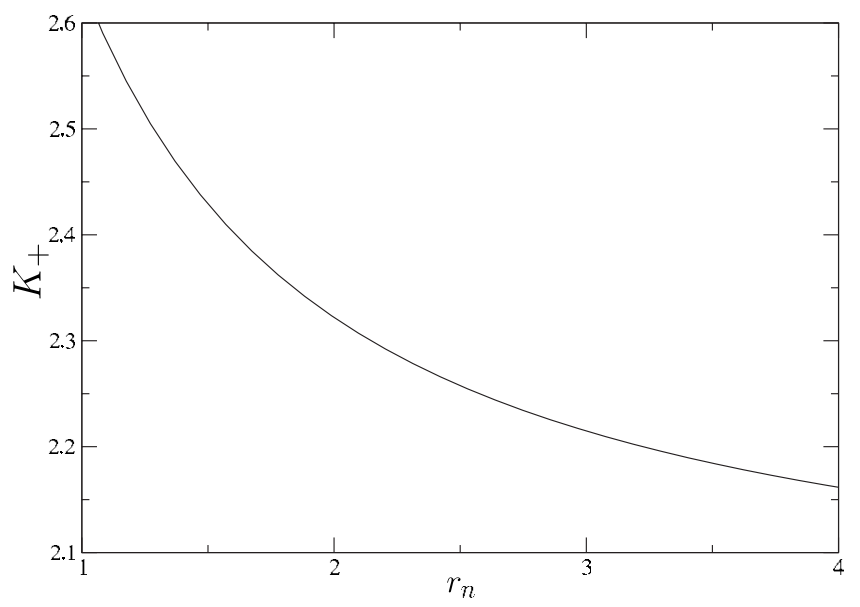

FIG. 4. The outer solution fixes the value of $K_{+}$as a function of the neck radius (which controls the drop volume). The maximal radius $r_{0}=3.38317 \cdots$ gives $K_{+}=2.17 \cdots$.

$$
\begin{aligned}
& \theta\left(s_{t}\right)=\pi, \\
& r\left(s_{t}\right)=0,
\end{aligned}
$$

where $s_{t}$ is the value of the arclength at the top. Two of these five boundary conditions serve as the definitions of $r_{n}$ and $s_{t}$, so that the remaining three boundary conditions fix the solution uniquely. The equations have been solved numerically.

Each value of $K_{+}$thus gives a solution with a different $r_{n}$, some of which are shown in Fig. 3. The numerically obtained relation between $K_{+}$and $r_{n}$ is depicted in Fig. 4. For the maximal neck radius $r_{n}=r_{0}=3.8317 \cdots$, introduced below, one finds $K_{+}=2.17 \cdots$.

The value of $r_{n}$ effectively sets the volume of the drop. Namely, the weight of the sessile drop is carried by the pressure exerted by the substrate on the contact area $\pi r_{n}^{2}$. The difference between the liquid and the gas pressures at $h=0$ is simply $K_{+}$, so we find

$$
K_{+} \pi r_{n}^{2}=2 \pi V_{+} \Rightarrow V_{+}=\frac{1}{2} K_{+} r_{n}^{2}
$$

where $V_{+}$is defined as the real volume divided by $2 \pi$, i.e.,

$$
V_{+}=\frac{1}{2 \pi} \int_{0}^{h_{\max }} d h \pi r^{2} .
$$

Note that to obtain the real liquid volume, one has to subtract the volume $V_{-}$of the gas pocket. However, $V_{-}$goes to zero in the limit of vanishing gas flow, as shown below.

\section{B. Outer "gas pocket" region}

In the "gas pocket" region, the profile $h(r)$ is no longer multivalued and we can express the curvature as

$$
\kappa=\frac{h^{\prime \prime}}{\left(1+h^{\prime 2}\right)^{3 / 2}}+\frac{h^{\prime}}{r\left(1+h^{\prime 2}\right)^{1 / 2}} .
$$

The solution is then specified by Eq. (24) with boundary conditions

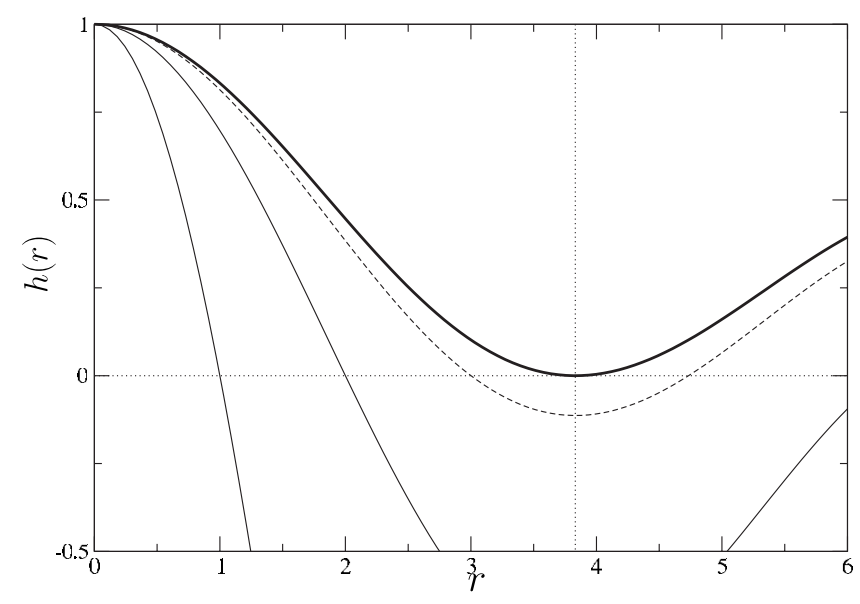

FIG. 5. Outer solutions for the gas pocket region (amplitudes normalized to unity). Thin solid lines correspond to $r_{n}=1,2$; the dashed line, corresponding to $r_{n}=3$, illustrates that $h$ would have to become negative to realize a neck radius larger than $r_{0}$. The heavy line shows the maximum possible $r_{n}=r_{0}$, corresponding to the first minimum of $J_{0}(r)$.

$$
\begin{aligned}
& h^{\prime}(0)=0, \\
& h\left(r_{n}\right)=0 .
\end{aligned}
$$

In Appendix A, we show that the solution can be written as an expansion,

$$
h(r)=-c_{-} \frac{J_{0}(r)-J_{0}\left(r_{n}\right)}{J_{0}\left(r_{n}\right)}+O\left(c_{-}^{3}\right),
$$

where $J_{0}(r)$ is a Bessel function of the first kind. Using furthermore that the curvature has to match the curvature of the inner solution $K_{-}$, and thus $K_{-}=h^{\prime \prime}\left(r_{n}\right)$, we can further simplify to

$$
h(r)=K_{-} \frac{J_{0}(r)-J_{0}\left(r_{n}\right)}{J_{0}^{\prime \prime}\left(r_{n}\right)}+O\left(K_{-}^{3}\right) .
$$

We see that the thickness scale of the gas pocket is set by the value of $K_{\text {. }}$. In the limit of vanishing flux, we expect this thickness to tend to zero, making $K_{-}$a small parameter. To find solution branches, it is crucial to go beyond linear order and to find the term of order $K_{-}^{3}$ in Eq. (40). The only quantity that is needed to perform the matching to Eq. (22), coming from the inner solution, is the slope $h_{-}^{\prime}\left(r_{n}\right)$. This calculation is done in Appendix A.

At this point we can already infer an upper bound on the possible values of $r_{n}$. Figure 5 shows the outer gas pocket solution (with normalized amplitude) for various values of $r_{n}$. The outer solutions are defined on the domain where $h(r) \geqslant 0$, hence the maximum possible neck radius is achieved when $J_{0}(r)$ has its first minimum at the maximal radius $r_{0}=3.8317 \cdots$ (vertical line). The corresponding solution is drawn with a heavy line.

A second remark is that $J_{0}^{\prime \prime}$ vanishes at $r \approx 1.852 \cdots$, so $K_{-}$ must become zero at this radius. At even smaller radii $J_{0}^{\prime \prime}$ turns negative, which yields negative values of $K_{-}$. However, the inner solution cannot reach large $h$ for negative $K_{-}$, 
which means that the matching procedure described here does not work. Dealing with this problem requires an additional matching region between the inner and outer (gas pocket) solution, the introduction of which is beyond the scope of this paper. We will simply stay away from $r_{n}$ $\approx 1.852$ and instead focus on radii close to the maximal value $r_{0} \approx 3.8317$, as detailed below.

\section{MATCHING THE ASYMPTOTIC REGIONS}

\section{A. Matching conditions}

We can now match the asymptotic regions by expressing Eqs. (20) and (21) in their original variables and expanding the outer solutions around $r=r_{n}$,

$$
\begin{aligned}
h_{\mathrm{out} \pm} & =\left.\frac{1}{2} h_{ \pm}^{\prime \prime}\right|_{r_{n}}\left(r-r_{n}\right)^{2}+\left.h_{ \pm}^{\prime}\right|_{r_{n}}\left(r-r_{n}\right), \\
h_{\mathrm{in} \pm} & =\frac{1}{2} K_{ \pm}\left(r-r_{n}\right)^{2}+\chi^{1 / 5} S_{ \pm}\left(r-r_{n}\right) .
\end{aligned}
$$

Therefore, the matching conditions become

$$
\begin{gathered}
K_{ \pm}=\left.h_{ \pm}^{\prime \prime}\right|_{r_{n}}, \\
\chi^{1 / 5} S_{ \pm}=\left.h_{ \pm}^{\prime}\right|_{r_{n}} .
\end{gathered}
$$

The conditions on the curvature were already taken into account when computing the outer profiles from Eq. (24). Typical values for $K_{+}$are of order unity, while the slope requires $\left.h_{+}^{\prime}\right|_{r_{n}}=0$ as $\chi \rightarrow 0$. This is why for the first outer solution we considered a perfectly nonwetting drop.

The - conditions are more subtle. The thickness of the gas pocket goes to zero asymptotically so that both $\left.h_{-}^{\prime \prime}\right|_{r_{n}}$ and $\left.h_{-}^{\prime}\right|_{r_{n}}$ will be small. In this case, the selection of the solution explicitly requires the slope condition, which we express as

$$
S_{-}=\frac{K_{-}}{\chi^{1 / 5}} \frac{\left.h_{-}^{\prime}\right|_{r_{n}}}{\left.h_{-}^{\prime \prime}\right|_{n}} \equiv-g\left(K_{-}, r_{n} ; \chi\right) .
$$

Together with Eq. (22), this closes the matching problem,

$$
f\left(K_{-}, K_{+}\right)=g\left(K_{-}, r_{n} ; \chi\right) .
$$

This equation indeed contains the three matching regions: $K_{+}$ implicitly depends on $r_{n}$ through the + outer solution, $f$ is determined by the inner solution, while $g$ follows from the outer solution.

\section{B. Bifurcation: Critical radius $r_{c}$}

For a given value of the flux $\chi$, we have reduced the problem to finding the intersections of the functions $f$ and $g$. This is sketched in Fig. 2, showing $f$ and $g$ for $\chi=10^{-7}$ and several values of $r_{n}$. Depending on the value of $r_{n}$, there can be two intersections, one intersection (when the curves are tangent), or no intersection. Each intersection corresponds to a stationary drop solution. This can be translated into a bifurcation diagram showing $K_{-}$versus $r_{n}$ (Fig. 7). For small

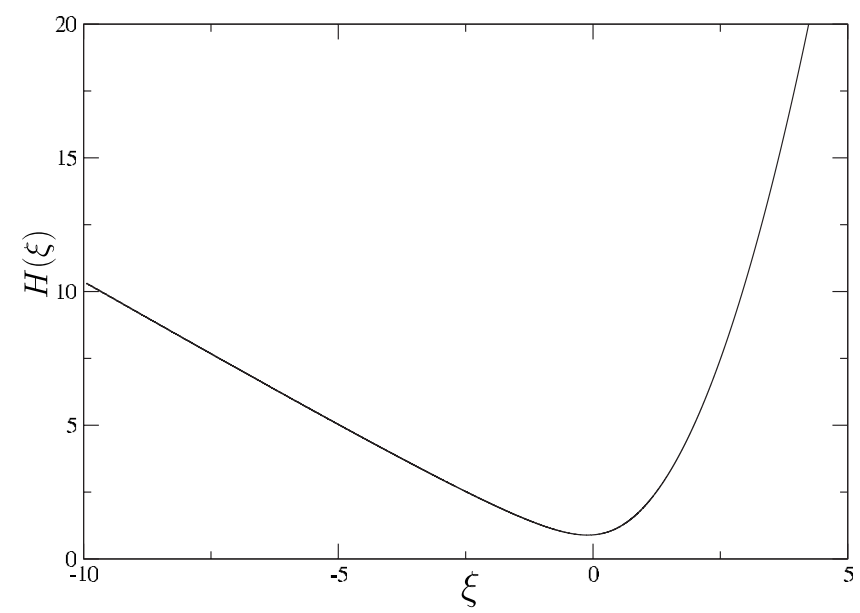

FIG. 6. The inner solution $H(\xi)$ obtained from numerical integration of Eq. (17), with $K_{+}=2.17$ and $K_{-}=0$. It will follow that these values correspond to the critical solution. The minimum value $H_{n} \approx 0.931$ determines the thickness of the neck (49).

radii there are two branches of solutions, corresponding to the two intersections, which merge at $r_{c}$. No stationary drop solutions exist for $r_{n}>r_{c}$.

We analyze the bifurcation in the limit of vanishing flux, $\chi \rightarrow 0$. We will show that

$$
r_{c}=r_{0}+O\left(\chi^{2 / 15}\right)
$$

so that the critical neck radius $r_{c}$ approaches the maximal radius $r_{0}$ in the limit of vanishing flux. To analyze the vicinity of the critical point, we introduce

$$
\tilde{r}=r_{0}-r_{n}
$$

At the same time we will find that $K_{-} \propto \chi^{1 / 15}$. This means that as the limit of $\chi$ going to zero is reached, $K_{-}=0$ and $r_{n}=r_{0}$, which implies $K_{+}=2.17$ according to Fig. 4. These two data fix the solution of Eq. (17) uniquely, and lead to the asymptotic profile shown in Fig. 6. From its minimum, one finds that

$$
h_{n} \approx 0.931 \chi^{2 / 5}
$$

in agreement with the scaling found by [1].

We now analyze the first correction to the solution as $\chi$ increases, but in the limit where $\chi, K_{-}, \tilde{r} \ll 1$. This can be done by considering the corresponding limit of the functions $f$ and $g$, cf. Fig. 2. Namely, the function $f$ approaches a constant, which is found numerically to be

$$
f \simeq f_{0}=1.12 \cdots .
$$

On the other hand, the asymptotic form of $g$ becomes

$$
g \simeq \chi^{-1 / 5} K_{-}\left(\widetilde{r}-g_{2} K_{-}^{2}\right) .
$$

The first term of Eq. (51) is found by expanding Eq. (40) for $r_{n}$ close to $r_{0}$, 


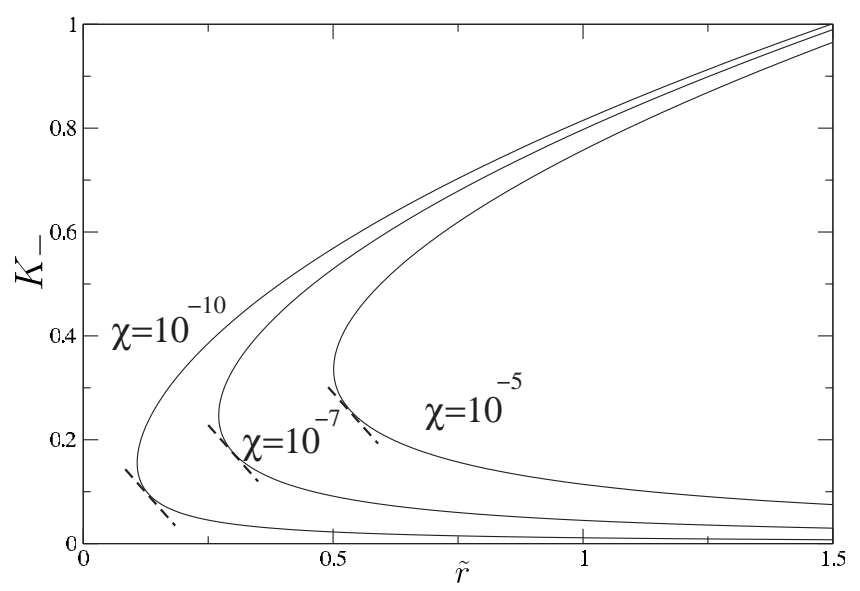

FIG. 7. The bifurcation diagram $\left(K_{-}, \widetilde{r}\right)$, derived from Eq. (54). Curves correspond to different values of the flux, $\chi$ $=10^{-5}, 10^{-7}, 10^{-10}$, revealing the weak dependence on $\chi$. The dashed lines represent perturbations $\delta \tilde{r}, \delta K_{-}=-\delta \widetilde{r} / c$, which are tangent to the solution curve. They represent marginal perturbations, separating stable from unstable solutions.

$$
\frac{\left.h_{-}^{\prime}\right|_{r_{n}}}{\left.h_{-}^{\prime \prime}\right|_{r_{n}}}=\frac{J_{0}^{\prime \prime}\left(r_{0}\right)\left(r-r_{n}\right)}{J_{0}^{\prime \prime}\left(r_{n}\right)}+O\left(K_{-}^{2}\right) \simeq-\widetilde{r}+O\left(K_{-}^{2}\right),
$$

where we used the property $J_{0}^{\prime}\left(r_{0}\right)=0$. We need to keep the $K_{-}^{2}$ term as it can become of the same order as $\widetilde{r}$. For details we refer to Appendix A, where we show that $g_{2}=1.486 \cdots$.

The matching condition $f=g$ [cf. Eq. (46)] is now reduced to a horizontal line intersecting a cubic function,

$$
f_{0}=\frac{K_{-}}{\chi^{1 / 5}}\left(\widetilde{r}-g_{2} K_{-}^{2}\right) .
$$

Solving for $\tilde{r}$, one finds

$$
\widetilde{r}\left(K_{-}, \chi\right)=\frac{\chi^{1 / 5} f_{0}}{K_{-}}+g_{2} K_{-}^{2},
$$

which has been plotted for different values of $\chi$ in Fig. 7. Thus for a given value of $r_{n}$ one finds two solution branches, which end at the critical value

$$
\tilde{r}_{c}=3\left(\frac{1}{4} g_{2} f_{0}^{2}\right)^{1 / 3} \chi^{2 / 15}+O\left(\chi^{4 / 15}\right)
$$

as claimed before. Note that the smallness of the power 2/15 makes $\widetilde{r}_{c}$ non-negligible. For typical experimental values of the flux, the critical point is thus substantially shifted with respect to the asymptotic value $r_{0}$.

Plugging this back into Eq. (54), one finds the value of $K_{-}$ at the critical point: $K_{-}^{(c)}=0.72 \chi^{1 / 15}$. But it follows from Eq. (40) that $h_{0}=h(0) \approx K_{-}\left[1-J_{0}\left(r_{0}\right)\right] / J_{0}^{\prime \prime}\left(r_{0}\right)$, and thus the maximum gap width is to leading order

$$
h_{0} \approx 2.52 \chi^{1 / 15} \text {. }
$$

This concludes the analysis of the stationary solutions, which are described by Eq. (54). At a given flow rate $\chi$, the critical neck radius is given by Eq. (55), which approaches the maximal value $r_{0}$ in the limit $\chi \rightarrow 0$.

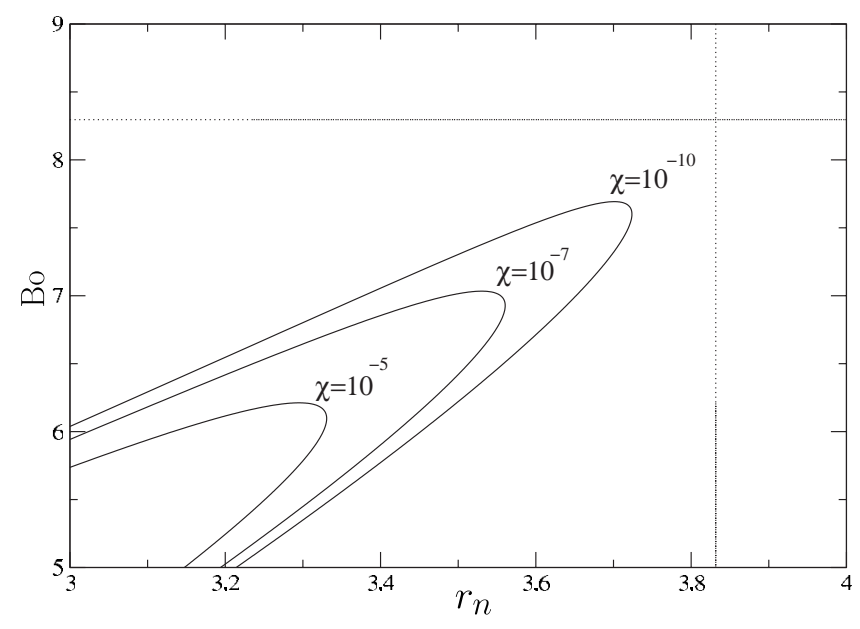

FIG. 8. The bifurcation diagram $\left(\mathrm{Bo}, r_{n}\right)$, for different values of the flux, $\chi=10^{-5}, 10^{-7}, 10^{-10}$. The maximum drop volume is attained slightly before the maximum radius $r_{c}$. This maximum coincides with the stability boundary shown as the dashed lines in Fig. 7. The dotted lines indicate the asymptotic limit for $\chi \rightarrow 0$.

\section{Drop volume: Bo versus $\boldsymbol{r}_{\boldsymbol{n}}$}

Experimentally the control parameter is the drop volume, measured by the Bond number (3), rather than $K_{-}$. As the size of the inner region is asymptotically small, one can compute the drop volume from the outer solutions. We already defined $V_{+}$as the volume of the sessile drop solution (35), i.e., without taking into account the gas layer. $V_{+}$is determined by the value of $r_{n}$. The real volume of the liquid is then obtained by subtracting the volume of the gas pocket,

$$
V_{-}\left(r_{n}, K_{-}\right)=\int_{0}^{r_{n}} d r h_{-}(r) \simeq \frac{1}{2} K_{-} r_{n}^{2}\left(\frac{J_{0}\left(r_{n}\right)-2 J_{1}\left(r_{n}\right) / r_{n}}{J_{0}\left(r_{n}\right)-J_{1}\left(r_{n}\right) / r_{n}}\right) .
$$

Note that this expression simplifies at $r_{0}$, because of the property $J_{1}\left(r_{0}\right)=0$.

The volume then becomes $V=2 \pi\left(V_{+}-V_{-}\right)$, yielding

$$
\mathrm{Bo}=\left(\frac{3}{2}\left(V_{+}-V_{-}\right)\right)^{2 / 3} \text {. }
$$

With this we can compute the Bond number for each value of $K_{+}$and $r_{n}$. Using furthermore the relation between $r_{n}$ and $K_{-}$ for stationary states (54), we can translate Fig. 7 into a bifurcation diagram in terms of Bo and $r_{n}$. This is plotted in Fig. 8, showing that stability of steady states is lost when the maximum drop volume is reached. This maximum occurs slightly before the maximum neck radius $r_{c}$. The resulting bifurcation diagram closely resembles the first branch of the numerical simulations in $[1,12]$.

\section{STABILITY BOUNDARY}

We now turn to the important question of which part of the solution branches shown in Fig. 7 is stable. Essentially, we find that the lower branch is linearly stable, while the upper is linearly unstable. Surprisingly, however, the mar- 
ginal point is not exactly at the maximum radius, but slightly before. We show that this marginal point corresponds to the stationary drop of maximum volume, as seen in Fig. 8.

\section{A. Stability limit}

To assess the stability of the drop solutions, we characterize the eigenmodes of the drop by infinitesimal variations in the neck position $r_{n}$. In principle, these deformations induce a flow inside the drop that is not taken into account in our static approximation for the outer solution. However, the question of whether solutions are stable is one of energetics, and thus can be addressed without explicitly computing the flow inside the liquid. In particular, at the marginal point, defined by a vanishing growth rate, there is no liquid flow to affect the pressure balance based on the flow in the gas alone.

We therefore consider infinitesimal variations in the neck position $\delta r_{n}$, and assess the corresponding change in levitation force $\delta F$. Since the pressure difference $\Delta p=p_{-}-p_{+}$ across the neck acts for $r<r_{n}$, this force reads

$$
F=\Delta p \pi r_{n}^{2}
$$

As mentioned, we will compute $\Delta p$ based on the gas flow alone. A marginal perturbation $\delta r_{n}$ occurs whenever the resulting levitation force is unchanged, $\delta F=F^{\prime} \delta r_{n}=0$, so that it still equilibrates the weight of the drop. Hence, we find the marginal condition

$$
\Delta p^{\prime}=-\frac{2 \Delta p}{r_{n}},
$$

where the prime denotes the derivative with respect to $r_{n}$. In order to produce the same levitation force, an increase in $r_{n}$ thus has to be compensated by a decrease of $\Delta p$. Had the pressure stayed constant, $F$ would be larger than the weight of the drop leading to the formation of a chimney and thus to instability. Similarly, pressures smaller than the marginal condition lead to a stable situation, giving the stability criterion

$$
\Delta p^{\prime}+\frac{2 \Delta p}{r_{n}}<0 .
$$

In the limit of small $\chi$, the pressure difference (25) is simply the difference of the curvatures,

$$
\Delta p=K_{+}-K_{-},
$$

so that stability requires

$$
K_{+}^{\prime}-K_{-}^{\prime}+\frac{2 \Delta p}{r_{n}}<0 .
$$

The derivative $K_{+}^{\prime}$ can be read off from Fig. 4, and is negative. Clearly, this has a stabilizing effect. The sign of $K_{-}^{\prime}$ can be inferred from the bifurcation diagram. The lower branch has a stabilizing contribution, while the upper branch is destabilizing. The location of the marginal point, however, depends on the numerical values of the three terms.
Taking the derivative of Eq. (34), we find

$$
K_{+}^{\prime}=\frac{2 V_{+}^{\prime}}{r_{n}^{2}}-\frac{2 K_{+}}{r_{n}} .
$$

Moreover, for vanishing flux $K_{-} \ll K_{+}$, hence we may replace $\Delta p \simeq K_{+}$, giving the stability criterion

$$
K_{-}^{\prime}>\frac{2 V_{+}^{\prime}}{r_{n}^{2}}
$$

Near the maximal radius $r_{n} \approx r_{0}$, the criterion for stability becomes

$$
K_{-}^{\prime}>c^{-1} \equiv \frac{2 V_{+}^{\prime}}{r_{0}^{2}}=0.92 \cdots .
$$

Indeed, the upper branch with $K_{-}^{\prime}<0$ is unstable, but the marginal point is not at the maximum radius $K_{-}^{\prime}=0$, but slightly before. This is indicated in Fig. 7 by the dashed lines, each having a slope of -0.92 . The - sign is because the figure uses $\tilde{r}=r_{0}-r_{n}$, while the derivative in Eq. (66) is taken with respect to $r_{n}$.

The maximum stable radius $\tilde{r}_{s}$ is found from Eq. (66),

$$
\frac{\chi^{1 / 5} f_{0}}{K_{-}^{2}}-2 g_{2} K_{-}=c,
$$

providing an equation for $K_{-}$at the stability boundary. This value of $K_{-}$is inconsistent with the asymptotic estimate $K_{-}$ $\approx \chi^{1 / 15}$ considered so far, indicating that the point where the solution exchanges stability is at a distance slightly larger from the critical point $r_{c}$. This means that $K_{-}$is smaller than expected (further down the lower branch; cf. Fig. 7). Thus the second term on the right of Eq. (67) is small compared to the other two, and we obtain

$$
K_{-}=\left(\frac{f_{0}}{c}\right)^{1 / 2} \chi^{1 / 10}
$$

If we evaluate Eq. (54) in the same limit, we finally obtain

$$
\tilde{r}_{s}=\left(f_{0} c\right)^{1 / 2} \chi^{1 / 10} .
$$

Thus for vanishing flux the maximum radius of stable solutions approaches $r_{0}$, but with an even smaller power than $r_{c}$. This scaling implies that $r_{s}<r_{c}<r_{0}$, as seen in Fig. 7.

We can show that this marginal point coincides with the maximum Bo shown in Fig. 8. This maximum is achieved when $d \mathrm{Bo} / d r_{n}=0$, which close to $r_{0}$ becomes

$$
V_{+}^{\prime}-\left(\frac{1}{2} K_{-} r_{n}^{2}\right)^{\prime} \simeq V_{+}^{\prime}-\frac{1}{2} r_{n}^{2} K_{-}^{\prime}=0 .
$$

Indeed, this is equivalent to Eq. (65) when $r_{n} \approx r_{0}$, as is the case for small enough flux $\chi$. This finding is again in agreement with the detailed numerical analysis by Lister et al. [12], in which the growth rate for the first branch was found to change sign at the maximum value of Bo.

\section{B. Linear stability analysis}

We now include dynamics in the stability analysis, once more assuming that the flow inside the drop can be ne- 
glected. At the end of this section, we discuss the range of parameters where this assumption is valid. We note first that an infinitesimal variation of the neck position, $\delta r_{n}=-\delta \widetilde{r}$, also induces a variation of the curvature, $\delta K_{-}$, and of the flux, $\delta \chi$. These three parameters are related through mass conservation of the liquid and the gas. The analysis is closed by a third equation coming from matching the dynamic inner region to the hydrostatic outer regions.

As before, we compute the volume of the liquid from the outer solutions as

$$
V_{\text {liquid }}=V_{+}\left(r_{n}\right)-V_{-}\left(r_{n}, K_{-}\right),
$$

which is exact up to asymptotically small corrections due to the inner region. The volume $V_{+}$is (numerically) determined by the value of $r_{n}$, while $V_{-}$was computed in Eq. (57). Since the liquid volume is strictly conserved, $\dot{V}_{\text {liquid }}=0$, one finds near $r_{0}$

$$
\delta K_{-}=-\frac{\delta \widetilde{r}}{c},
$$

where the constant $c$ has been defined by Eq. (66). Relation (72) expresses the fact that when $r_{n}$ increases, increasing $V_{+}$, the volume of the gas pocket has to increase by a similar amount to keep the liquid volume constant. This is achieved by an increase of $K_{-}$.

Mass conservation of the gas is described by continuity (7), which can be integrated to

$$
r h \bar{u}=\Gamma(r)-\frac{\partial}{\partial t} \int_{0}^{r} d r r h .
$$

The second term on the right-hand side can be identified as the rate of change of gas pocket volume $\dot{V}_{-}$, which we will write as $-V_{+}^{\prime} \delta \dot{r}$. This change absorbs part of the injected air, decreasing the flux passing across the neck. Considering the radius somewhere inside the neck region, $r \approx r_{n}$, the equation can be simplified to [using Eq. (11) and $h^{\prime} \ll 1$ ]

$$
h^{3} h^{\prime \prime \prime}=\chi+\delta \chi,
$$

where the variation of the flux reads

$$
\delta \chi=\frac{r_{0}}{24 c} \delta \dot{\tilde{r}} .
$$

The matching condition (54) closes the dynamical system, taking into account the dependencies (72) and (75). The marginal case $\delta \chi=0$ corresponds to a curve tangent to any of the lines $\widetilde{r}\left(K_{-}\right)$shown in Fig. 7. Since in addition the slope of such a tangent curve must be $-c^{-1}$ according to Eq. (72), this uniquely fixes a point on any of the lines at constant $\chi$. The critical tangent curve was already drawn dashed in Fig. 7 , based on the analysis of the previous section. Below this point, on the lower branch, solutions are stable; above they are unstable.

Formally, the growth rate of perturbations is computed by writing

$$
\delta \dot{\tilde{r}}=\sigma \delta \widetilde{r} .
$$

Now using Eqs. (72) and (75), and the first variation of Eq. (54), one finds

$$
\sigma=\frac{24}{c r_{0}}\left(\frac{\partial \widetilde{r}}{\partial \chi}\right)^{-1}\left[\frac{\partial \widetilde{r}}{\partial K_{-}}+c\right] .
$$

The partial derivatives are to be evaluated from Eq. (54). The maximum stable radius is found by the condition $\sigma=0$. This indeed gives the same stability boundary as Eq. (65), which was based on a global force balance [note that $\partial \widetilde{r} / \partial K_{-}=$ $\left.-\left(K_{-}^{\prime}\right)^{-1}\right]$.

Let us conclude this section by discussing the effect of flow inside the drop, which was neglected in the above analysis. As noted by Lister et al. [12], one needs to compare the relaxation time $\sigma^{-1}$ of the gas flow to the viscous relaxation time scale of the drop,

$$
t_{\text {drop }}=\frac{\ell_{c} \eta_{\text {drop }}}{\gamma},
$$

which for water is of the order of $10^{-5} \mathrm{~s}$. Throughout our analysis we used the gas viscosity $\eta_{\text {gas }}$ to rescale all variables, so that the gas relaxation time becomes

$$
t_{\mathrm{gas}}=\frac{\ell_{c} \eta_{\mathrm{gas}}}{\sigma \gamma}=\frac{t_{\mathrm{drop}}}{\sigma \lambda},
$$

where $\lambda=\eta_{\text {drop }} / \eta_{\text {gas }}$.

As $\sigma$ can be arbitrarily small upon approaching the instability, there is always a region where we find that $t_{\text {gas }} \ll t_{\text {drop }}$, so that the flow inside the drop can indeed be neglected. To estimate the range of validity away from the marginal point, we evaluate $\sigma$ at the maximum radius $\widetilde{r}_{c}$, where $\partial \widetilde{r} / \partial K_{-}=0$ (cf. Fig. 7). At this point we find

$$
\frac{t_{\text {gas }}}{t_{\text {drop }}} \approx 0.05 \lambda^{-1} \chi^{-13 / 15} .
$$

Typical values for Leidenfrost drops can be estimated (see Sec. VIII) as $\chi \sim 10^{-4}$ and $\lambda \sim 10^{2}$, so that the ratio of time scales $\approx 1.5$ when the maximum radius is reached. Hence, liquid flow begins to be relevant for these parameter values. The range of validity of our theory will of course be larger when the flow rate is reduced further. We wish to note, however, that for water viscous time scales are very fast compared to the eigenfrequency of the drop, so that inertial effects may also come into play.

\section{NUMERICAL TESTS}

\section{A. Nonlinear dynamical behavior}

We begin with a simulation of the full axisymmetric Stokes problem, using a boundary integral method [23], which has the advantage that it tracks the interface with high precision. The idea is to regard the interface as a continuous distribution of point forces, which point in the direction of the normal and whose strength is proportional to the mean curvature. Since for Stokes flow one knows the Green function giving the velocity field resulting from a point force, one 


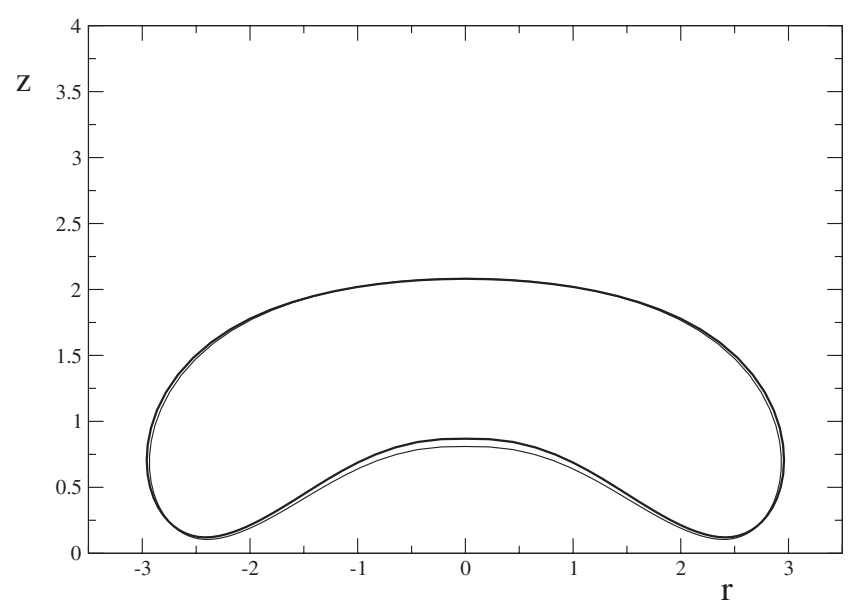

FIG. 9. Boundary integral simulation of a drop with parameters $\Gamma=0.02, \mathrm{Bo}=4.2$, and $\lambda=100$. The drop relaxes toward a stable state, which is drawn as the heavy line.

can write the velocity anywhere in space as an integral over the free surface. In an axisymmetric situation, the angle integral can be performed, so the remaining integration is onedimensional.

External flow sources can simply be added; in the present case we take the gas flow as a point source of strength $Q$ situated at the origin on the solid plate that bounds the flow. For this a simple exact solution is available [24]. Likewise for the Green function one must take into account the presence of a no-slip wall. This is possible using the method of images [25], and the resulting boundary integral formulation has been applied successfully to the motion of drops relative to a wall [26]. If, as in our case, the viscosity of the drop is different from that of the surrounding, one must account for the stress mismatch across the interface. This can be done at the cost of introducing another integral over the velocity on the interface into the equation, which turns the equation for the velocity field into an integral equation. After solving this equation for a given interface shape, the thus computed velocity field can be used to advance the interface.

We follow closely an earlier implementation of the boundary integral method, used, for example, in the coalescence of two drops inside another fluid [27]. The only significant difference is that the free-space Green function has been replaced by the half-space Green function, bounded by a wall. We tested the code by comparing to an exact solution of a sphere moving perpendicular to a wall [28]. This is realized in the limit of a very small drop, or of very large drop viscosity, so that there is hardly any deformation. The agreement was good, but significant deviations occurred when the gap between the wall and the drop was smaller than $5 \%$ of the drop radius. At present, we do not know the origin of this numerical problem, which prohibits us from investigating the asymptotic limit of very small gap spacings. Instead, we report on simulations at moderate gap spacings, which show the nonlinear stages of chimney formation, not captured by our linear stability analysis.

Figure 9 shows a viscous drop that is slightly smaller than the stability boundary. Starting from a configuration shown as the light curve, it relaxes toward a stationary stable state

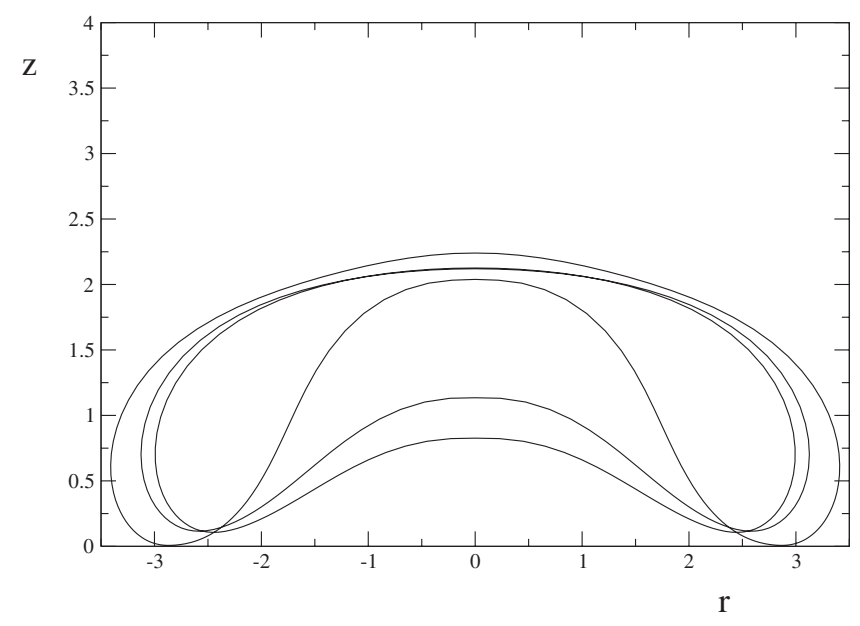

FIG. 10. The same as Fig. 9, but with a slightly larger $\mathrm{Bo}=4.4$. The air bubble under the center of the drop lifts up to form a chimney. The time interval between the profiles is $\Delta t=3000$, in units of $\ell_{c} \eta_{\mathrm{gas}} / \gamma$.

(heavy line). For a Bond number that is just slightly larger, the same initial condition leads to a rising gas bubble in the center of the drop; see Fig. 10. A thin film forms between the rising gas bubble and the top of the drop, which drains slowly. As seen in Fig. 9, the neck radius is $r_{n} \approx 2.5$, giving $\chi=0.015$. On the basis of our asymptotic theory (69), a rough estimate of the stability boundary gives $r_{s} \approx 3.2$, somewhat larger than the expected value of 2.5. In the following section it will become clear that such a difference is consistent with the slow convergence to the asymptotic regime.

\section{B. Lubrication approximation}

To test the bifurcation scenario in more detail, we resort to direct numerical simulation of the lubrication equation. Due to the overhang of the drop, we separate the upper part of the drop and the lower part of the drop at the maximum radius, $r_{\max }$, defined by the point $\left|h^{\prime}\right|=\infty$. The upper part is solved as described in Sec. IV A, and for the lower part of the drop we use

$$
\begin{gathered}
\kappa=\frac{h^{\prime \prime}}{\left(1+h^{\prime 2}\right)^{3 / 2}}+\frac{h^{\prime}}{r\left(1+h^{\prime 2}\right)^{1 / 2}}, \\
\chi=h^{3}\left(\kappa^{\prime}+h^{\prime}\right) .
\end{gathered}
$$

This describes both the inner and outer regions in the lower part of the interface, while we have conveniently taken the rate of injection $\Gamma(r) / r$ to be constant for all $r$. Boundary conditions for this third-order equation are

$$
\begin{gathered}
h^{\prime}(0)=0, \\
h^{\prime}\left(r_{\max }\right)=\infty, \\
\kappa\left(r_{\max }\right)=\kappa_{\text {patch }},
\end{gathered}
$$

where $\kappa_{\text {patch }}$ is the curvature at the point where the upper and lower solutions are patched. A one-parameter family of solu- 


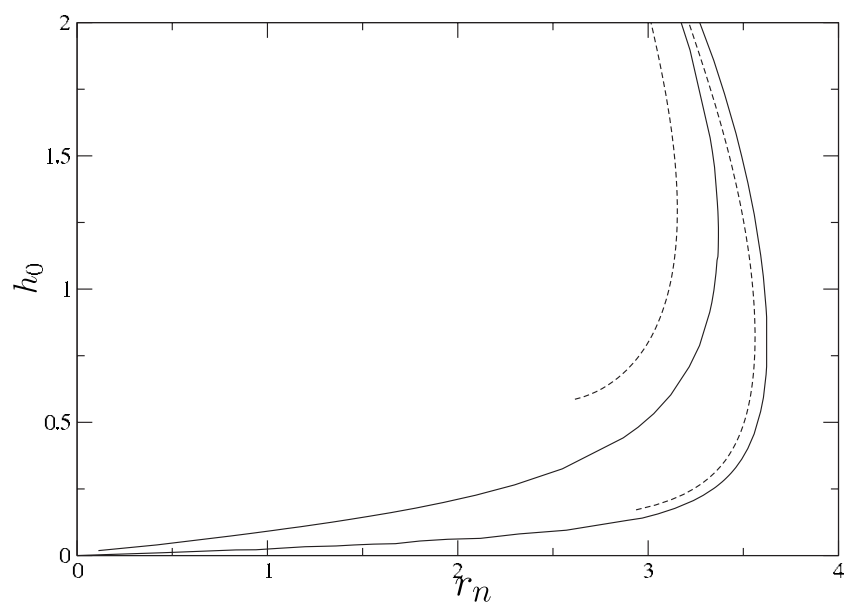

FIG. 11. Bifurcation diagram $h_{0}$ vs $r_{n}$ for $\chi=10^{-4}$ and $10^{-7}$. Smaller $\chi$ yield larger radii. Solid lines were obtained from numerical solution of the lubrication equation (82). Dashed lines correspond to asymptotic theory (54).

tions is obtained through variation of the upper part of the drop. It was shown in [1] that this procedure provides drop solutions that are quantitatively accurate.

The numerically obtained drop profiles are conveniently characterized by the position of the neck, $r_{n}$, and the gap below the center of the drop, $h_{0}$. Numerical results for the solution branches are shown as solid lines in Fig. 11 for two values of the flux. $\chi=10^{-4}$ is a typical experimental value encountered for Leidenfrost drops, while $\chi=10^{-7}$ illustrates the convergence toward the asymptotic limit. As predicted, there is a critical radius beyond which no stationary solutions exist. The asymptotic predictions shown in Fig. 7 have been translated to the dashed lines of Fig. 11. These are obtained from Eq. (54), where $K_{-}$was computed from $h_{0}$ using Eq. (40). Good quantitative agreement is achieved for small enough values of the flux.

Finally, we determined the critical radius $r_{c}$ for a range of values of the flux $\chi$. Figure 12 shows how the numerical values (dots) indeed approach the asymptotic prediction (solid line) in the limit of vanishing flux. Due to the very small powers $\chi^{2 / 15}$, the convergence toward $r_{0}=3.8317 \ldots$ is extremely slow (horizontal line). As a consequence, the correction with respect to this asymptotic value will be significant for typical experimental values of the flux.

\section{DISCUSSION}

Owing to the smallness of the neck region (49), we can make the simplification that the pressure inside the gas pocket below the drop is constant (Fig. 1). This pressure is larger than the atmospheric pressure and provides the force required to levitate the drop. Matching the pressure difference across the neck with the viscous flow then provides the bifurcation diagram of Fig. 7, yielding a critical neck radius $r_{c}$. In the limit of vanishing flux, the critical radius approaches $r_{0}=3.8317 \ldots$. This value arises because it is the first minimum of the function characterizing the shape of the gas pocket, which is the Bessel function $J_{0}(r)$. For larger $r_{n}$,

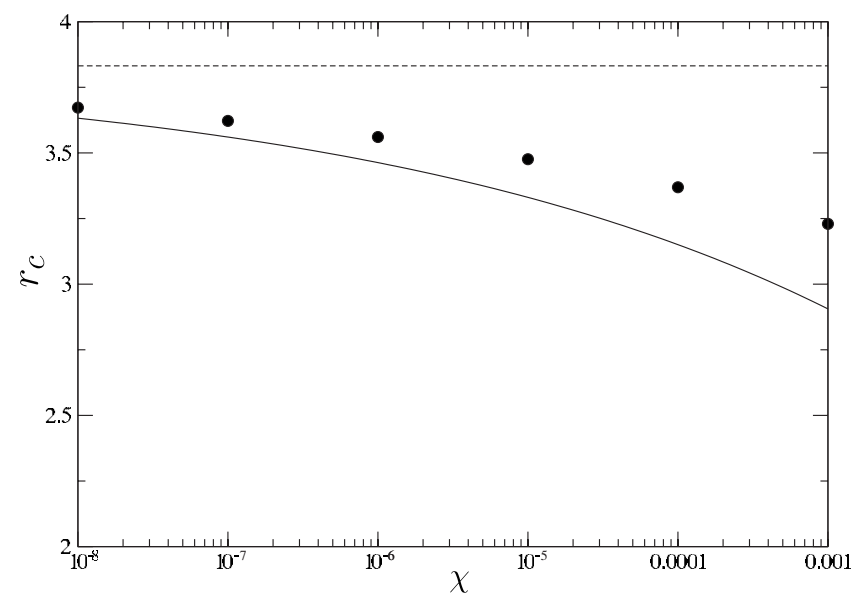

FIG. 12. Critical radius $r_{c}$ as a function of the flux $\chi$. The numerical values obtained from the lubrication equation (dots) indeed approach the theoretical prediction (55) (solid line). The dashed line indicates the asymptotic value $r_{0}=3.8317 \ldots$.

the gas pocket shape would need to become negative, which is of course not allowed.

Experimentally, the size of the drop is measured by looking at the drop from above. This measurement provides the maximum radius $r_{\max }$ rather than the neck radius; cf. Fig. 1. For large puddles the difference between $r_{\max }$ and $r_{n}$ approaches $\sqrt{2}-\operatorname{arc} \cosh \sqrt{2} \approx 0.53$. For drop sizes relevant here we confirmed numerically that $r_{\max }-r_{n} \approx 0.52$. Combined with Eq. (55), we thus find

$$
r_{\max , \mathrm{st}} \approx 4.35-1.02 \chi^{1 / 10}
$$

for the boundary of stability, expressed in terms of the capillary length. Typical experimental values of $\chi$ can be extracted using Eq. (49), and typical experiments yield $h_{n}$ $\approx 100 \mu \mathrm{m}$, obtained from diffraction data [5]. This gives $\chi$ $\approx 10^{-4}$, and thus $r_{\text {max }, \text { st }} \approx 3.95$, to be compared with reported experimental values of $4.0 \pm 0.2$ [5,11]. A similar estimate of $\chi$ is obtained by considering the latent heat of evaporation [5]. Furthermore, our boundary integral simulations show that the nonlinear dynamics for larger drops lead to the formation of chimneys, as observed experimentally. We are therefore confident that the analysis in terms of Stokes flow provides an accurate description of this instability.

Let us now return to the argument put forward in $[5,11]$, relating chimney formation to the Rayleigh-Taylor instability. The latter occurs when a layer of fluid is suspended above another fluid of lower density, so that the system tends to destabilize due to buoyancy forces. Surface tension opposes this effect, so that the instability occurs at long wavelengths only. Biance et al. $[5,11]$ propose that levitated drops remain stable as long as axisymmetric perturbations that fit inside the drop are stable with respect to this buoyancydriven instability.

For an infinitely extended liquid film, one finds that $J_{0}(k r)$ are axisymmetric eigenmodes, with the stability criterion $k$ $>1$. While the Bessel function does not have a well-defined period, the maximum drop size was estimated in [11] by the first minimum of the mode with $k=1$, occurring at $r_{0}$. In 
hindsight, our results justify this choice of taking the minimum of $J_{0}(r)$ as the stability boundary, provided that it is identified with the neck radius, rather than with $r_{\text {max }}$. With this connection, our results reduce to the Rayleigh-Taylor argument in the limit of vanishing gas flow, showing that the balance between buoyancy and surface tension provides the right mechanism. The effect of the gas flow is to reduce slightly the range of stable solutions (86).

We close the discussion by comparing our results once more with the numerical findings in $[1,12]$, obtained for drops levitating above a curved mould. The bifurcation scenario for such a curved substrate was found to be much richer than the single fold considered in the present paper. In particular, a new set of solutions emerges above $r_{n} \approx 7$, displaying multiple folds. We believe these new solutions result from the curvature of the mould, allowing the substrate to "touch" the outer solution at new locations; cf. Fig. 5. As the next extremum of $J_{0}(r)$ after $r_{0}=3.83 \ldots$ is found at $r$ $=7.02 \ldots$, it may be worthwhile to pursue this connection in more detail.

\section{ACKNOWLEDGMENTS}

We thank John Lister for pointing out the importance of the liquid viscosity for the time scale of chimney formation and for sharing his work prior to publication. J.H.S. acknowledges support from a Marie Curie European Action FP6 (Grant No. MEIF-CT-2006-025104).

\section{APPENDIX A: GAS POCKET SOLUTION}

In this appendix, we expand the gas pocket solution for small amplitudes and compute the constant $g_{2}$. We consider the equation

$$
\frac{h^{\prime \prime}}{\left(1+h^{\prime 2}\right)^{3 / 2}}+\frac{h^{\prime}}{r\left(1+h^{\prime 2}\right)^{1 / 2}}+h=c_{-},
$$

with boundary conditions

$$
\begin{aligned}
& h^{\prime}(0)=0, \\
& h\left(r_{n}\right)=0 .
\end{aligned}
$$

This is equivalent to solving

$$
\frac{y^{\prime \prime}}{\left(1+y^{\prime 2}\right)^{3 / 2}}+\frac{y^{\prime}}{r\left(1+y^{\prime 2}\right)^{1 / 2}}+y=0
$$

with boundary conditions

$$
\begin{gathered}
y^{\prime}(0)=0, \\
y\left(r_{n}\right)=-A,
\end{gathered}
$$

where $A=c_{-}$We expand in $A$,

$$
y(r)=A y_{1}(r)+A^{3} y_{3}(r)+O\left(A^{5}\right) .
$$

This yields a hierarchy of equations

$$
\begin{gathered}
y_{1}^{\prime \prime}+\frac{y_{1}^{\prime}}{r}+y_{1}=0, \\
y_{3}^{\prime \prime}+\frac{y_{3}^{\prime}}{r}+y_{1}=\frac{3}{2} y_{1}^{\prime 2} y_{1}^{\prime \prime}+\frac{1}{2 r} y_{1}^{\prime 3},
\end{gathered}
$$

with boundary conditions

$$
\begin{gathered}
y_{1}^{\prime}(0)=0, \\
y_{1}\left(r_{n}\right)=-1, \\
y_{3}^{\prime}(0)=0, \\
y_{3}\left(r_{n}\right)=0 .
\end{gathered}
$$

The first equation gives $y_{1}(r)=-J_{0}(r) / J_{0}\left(r_{n}\right)$, which can be inserted into the right-hand side of the equation for $y_{3}(r)$.

To compute the constant $g_{2}$, we require the ratio $y^{\prime}\left(r_{0}\right) / y^{\prime \prime}\left(r_{0}\right)$. In terms of the expansion,

$$
\frac{y^{\prime}\left(r_{0}\right)}{y^{\prime \prime}\left(r_{0}\right)}=A^{2} y_{3}^{\prime}\left(r_{0}\right)+O\left(A^{4}\right),
$$

where we used the properties $y_{1}\left(r_{0}\right)^{\prime}=0$ and $y_{1}^{\prime \prime}\left(r_{0}\right)=-y_{1}\left(r_{0}\right)$ $=1$. Comparing to Eq. (51), we simply find

$$
g_{2}=y_{3}^{\prime}\left(r_{0}\right) \text {. }
$$

We obtained this value numerically by solving the ODE for $y_{3}$, for which we numerically obtained $g_{2}=1.486 \cdots$.
[1] L. Duchemin, J. R. Lister, and U. Lange, J. Fluid Mech. 533, 161 (2005).

[2] J. G. Leidenfrost, De Aquae Communis Nonnullis Qualitibus Tractatus, Part 2 (Hermannus Ovenius, Duisburg, 1756).

[3] N. J. Holter and W. R. Glasscock, J. Acoust. Soc. Am. 24, 682 (1952).

[4] M. A. Goldshtick, V. M. Khanin, and V. G. Ligai, J. Fluid Mech. 166, 1 (1986).

[5] A. L. Biance, C. Clanet, and D. Quéré, Phys. Fluids 15, 1632 (2003).

[6] H. Linke, B. J. Alemán, L. D. Melling, M. J. Taormina, M. J.
Francis, C. C. Dow-Hygelund, V. Narayanan, R. P. Taylor, and A. Stout, Phys. Rev. Lett. 96, 154502 (2006).

[7] Y. Couder, S. Protière, E. Fort, and A. Boudaoud, Nature 437, 208 (2005).

[8] T. Gilet, N. Vandewalle, and S. Dorbolo, Phys. Rev. E 76, 035302(R) (2007).

[9] C. Wagner, Y. Amarouchene, Daniel Bonn, and J. Eggers, Phys. Rev. Lett. 95, 164504 (2005).

[10] E. Hervieu, N. Coutris, and C. Boichon, Nucl. Eng. Des. 204, 167 (2001).

[11] A. L. Biance, Ph.D. thesis, University of Paris VI (2003). 
[12] J. R. Lister, A. B. Thompson, A. Perriot, and L. Duchemin, J. Fluid Mech. 617, 167 (2008).

[13] L. H. J. Watchers, H. Bonne, and H. J. van Nouhuis, Chem. Eng. Sci. 21, 923 (1966).

[14] K. Adachi and R. Takaki, J. Phys. Soc. Jpn. 53, 4184 (1984).

[15] R. Takaki and K. Adachi, J. Phys. Soc. Jpn. 54, 2462 (1985).

[16] N. Tokugawa and R. Takaki, J. Phys. Soc. Jpn. 63, 1758 (1994).

[17] D. E. Strier, A. A. Duarte, H. Ferrari, and G. Mindlin, Physica A 283, 261 (2000).

[18] A. Snezhko, E. Ben Jacob, and I. S. Aranson, New J. Phys. 10, 043034 (2008).

[19] N. Yoshiyasu, K. Matsuda, and R. Takaki, J. Phys. Soc. Jpn. 65, 2068 (1996).
[20] G. K. Batchelor, An Introduction to Fluid Dynamics (Cambridge University Press, Cambridge, UK, 1967).

[21] A. F. Jones and S. D. R. Wilson, J. Fluid Mech. 87, 263 (1978).

[22] J. R. Lister, N. F. Morrison, and J. M. Rallison, J. Fluid Mech. 552, 345 (2006).

[23] J. M. Rallison and A. Acrivos, J. Fluid Mech. 89, 191 (1978).

[24] J. Happel and H. Brenner, Low Reynolds Number Hydrodynamics (Martinus Nijhoff, The Hague, 1983).

[25] J. R. Blake, Proc. Cambridge Philos. Soc. 70, 303 (1971).

[26] C. Pozrikidis, J. Fluid Mech. 215, 331 (1990).

[27] J. Eggers, J. R. Lister, and H. A. Stone, J. Fluid Mech. 401, 293 (1999).

[28] E. Bart, Chem. Eng. Sci. 23, 193 (1968). 